\title{
Aminocyclopentadienyl Ruthenium Complexes as Racemization Catalysts for Dynamic Kinetic Resolution of Secondary Alcohols at Ambient Temperature
}

Jun Ho Choi, Yoon Kyung Choi, Yu Hwan Kim, Eun Sil Park, Eun Jung Kim, Mahn-Joo Kim, * and

Jaiwook Park*

National Research Laboratory of Chirotechnology, Department of Chemistry, Division of Molecular and Life Sciences, Pohang University of Science and Technology, Pohang, Kyungbuk 790-784, Korea

E-mail: pjw@postech.ac.kr, mjkim@postech.ac.kr.

\section{Supporting Information}

Table of Contents

Synthetic Procedures and Characterization of Ruthenium Complexes 5-13

Characterization of the Chiral Acetates produced in the DKR of Various Alcohols

References

S-11
Page

S-2

S-6 


\section{Synthetic Procedures and Characterization of Ruthenium Complexes 5-13.}

$\left[\mathbf{2 , 3}, \mathbf{4 , 5}-\mathrm{Ph}_{4}\left(\eta^{5}-\mathbf{C}_{4} \mathbf{C N H}^{n} \mathbf{P r}\right)\right] \mathbf{R u}(\mathbf{C O})_{2} \mathbf{C l}(\mathbf{5})$ : By heating a solution of $\mathrm{Ru}_{3}(\mathrm{CO})_{12}(0.60 \mathrm{~g}, 0.9 \mathrm{mmol})$, the imine $(1.00 \mathrm{~g}, 2.4 \mathrm{mmol})$, and dry 2-propanol $(270 \mu \mathrm{L}, 3.5 \mathrm{mmol})$ in chloroform $(24 \mathrm{~mL})$ at $90{ }^{\circ} \mathrm{C}$ for $48 \mathrm{~h}, 5$ was obtained in $34 \%$ yield (490 mg). Mp: 189-190 ${ }^{\circ} \mathrm{C}$ (dec.); ${ }^{1} \mathrm{H}$ NMR (300 MHz, $\mathrm{CDCl}_{3}$ ): $\delta$ 7.59-6.90 (m, 20H), $4.22(\mathrm{t}, J=5.4 \mathrm{~Hz}, 1 \mathrm{H}), 2.68\left(\mathrm{dt}, J_{l}=5.4 \mathrm{~Hz}, J_{2}=7.2 \mathrm{~Hz}, 2 \mathrm{H}\right), 1.23(\mathrm{~m}, 2 \mathrm{H}), 0.57(\mathrm{t}$, $J=7.2 \mathrm{~Hz}, 3 \mathrm{H}) ;{ }^{13} \mathrm{C} \mathrm{NMR}\left(75 \mathrm{MHz}, \mathrm{CDCl}_{3}\right): \delta 198.9,144.1,133.9,132.4,131.0,129.4,129.1,128.7$, 128.2, 110.1, 101.9, 83.5, 47.3, 24.3, 11.5; IR $\left(\mathrm{KBr}, \mathrm{cm}^{-1}\right): \mathrm{v}(\mathrm{CO})=2026,1970 ; \mathrm{MS}(\mathrm{FAB}): \mathrm{m} / \mathrm{z}(\%)$ : 620 (4) $\left[\mathrm{M}^{+}+1\right], 584$ (37), 563 (100), 526 (75). Anal. Calcd for $\mathrm{C}_{34} \mathrm{H}_{28} \mathrm{NO}_{2} \mathrm{ClRu}$ : C, 65.96; H, 4.56; N, 2.26. Found: C, 65.74; H, 4.19; N, 2.11 .

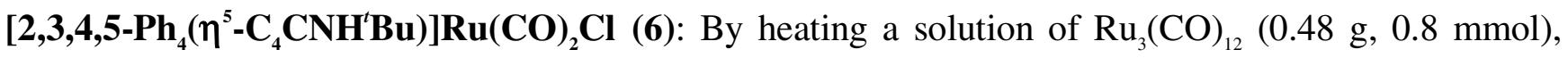
the imine $(1.00 \mathrm{~g}, 2.3 \mathrm{mmol})$, and dry 2-propanol $(260 \mu \mathrm{L}, 3.4 \mathrm{mmol})$ in chloroform $(28 \mathrm{~mL})$ at $90{ }^{\circ} \mathrm{C}$ for $72 \mathrm{~h}, 6$ was obtained in $24 \%$ yield $(359 \mathrm{mg})$. Mp: $182-184{ }^{\circ} \mathrm{C}$ (dec.); ${ }^{1} \mathrm{H}$ NMR (300 MHz, $\mathrm{CDCl}_{3}$ ): $\delta$ 7.56-6.88 (m, 20H), $4.31(\mathrm{~s}, 1 \mathrm{H}), 0.95(\mathrm{~s}, 9 \mathrm{H}) ;{ }^{13} \mathrm{C} \mathrm{NMR}\left(75 \mathrm{MHz}, \mathrm{CDCl}_{3}\right): \delta 198.8,145.7,134.9$, 132.6, 131.7, 131.3, 129.3, 128.6, 128.1, 110.1, 102.6, 84.4, 54.6, 31.9; IR $\left(\mathrm{KBr}, \mathrm{cm}^{-1}\right): v(\mathrm{CO})=2060$, 1992; MS (FAB): $m / z(\%): 634(12)\left[\mathrm{M}^{+}+1\right], 598$ (64), 577 (93), 542 (100). Anal. Calcd for $\mathrm{C}_{35} \mathrm{H}_{30} \mathrm{NO}_{2} \mathrm{ClRu}: \mathrm{C}, 66.40 ; \mathrm{H}, 4.78 ; \mathrm{N}, 2.21$. Found: C, 66.04; H, 4.64; N, 2.09. 
[2,5- $\left.\mathrm{Me}_{2}-\mathbf{3}, \mathbf{4}-\mathrm{Ph}_{2}\left(\eta^{5}-\mathrm{C}_{4} \mathrm{CNH}^{i} \mathrm{Pr}\right)\right] \mathbf{R u}(\mathrm{CO})_{2} \mathrm{Cl}$ (7): By heating a solution of $\mathrm{Ru}_{3}(\mathrm{CO})_{12}(0.09 \mathrm{~g}, 0.1$ $\mathrm{mmol})$, the imine $(0.10 \mathrm{~g}, 0.3 \mathrm{mmol})$, and dry 2-propanol $(40 \mu \mathrm{L}, 0.5 \mathrm{mmol})$ in chloroform $(4 \mathrm{~mL})$ at $90{ }^{\circ} \mathrm{C}$ for $20 \mathrm{~h}, 7$ was obtained in $64 \%$ yield $(105 \mathrm{mg})$. Mp: $164-165{ }^{\circ} \mathrm{C}$ (dec.); ${ }^{1} \mathrm{H}$ NMR (300 MHz, $\left.\mathrm{CDCl}_{3}\right): \delta$ 7.27-7.15 (m, 10H), 4.0-3.94 (m, 2H), $1.99(\mathrm{~s}, 6 \mathrm{H}), 1.37(\mathrm{~d}, J=5.61 \mathrm{~Hz}, 6 \mathrm{H}) ;{ }^{13} \mathrm{C}$ NMR $(75$ $\left.\mathrm{MHz}, \mathrm{CDCl}_{3}\right): \delta 198.9,140.4,131.9,130.7,128.5,128.3,102.9,75.5,45.8,25.9,11.0 ; \mathrm{IR}\left(\mathrm{KBr}, \mathrm{cm}^{-1}\right):$ $v(\mathrm{CO})=2016,1966 ; \mathrm{MS}(\mathrm{FAB}): \mathrm{m} / z(\%): 496(9)\left[\mathrm{M}^{+}+1\right], 460$ (45), 439 (100), 402 (76). Anal. Calcd for $\mathrm{C}_{24} \mathrm{H}_{24} \mathrm{NO}_{2} \mathrm{ClRu}: \mathrm{C}, 58.24 ; \mathrm{H}, 4.89 ; \mathrm{N}, 2.83$. Found: C, 57.92; H, 4.83; N, 2.87.

[2,5-Me $\left.-\mathbf{- 3 , 4 - \mathrm { Ph } _ { 2 }}\left(\eta^{5}-\mathrm{C}_{4} \mathrm{CNHPh}\right)\right] \operatorname{Ru}(\mathrm{CO})_{2} \mathrm{Cl}(\mathbf{8})$ : By heating a solution of $\mathrm{Ru}_{3}(\mathrm{CO})_{12}(0.15 \mathrm{~g}, 0.2$ $\mathrm{mmol})$, the imine $(0.20 \mathrm{~g}, 0.6 \mathrm{mmol})$, and dry 2-propanol $(67 \mu \mathrm{L}, 0.9 \mathrm{mmol})$ in chloroform $(8 \mathrm{~mL})$ at $90{ }^{\circ} \mathrm{C}$ for $12 \mathrm{~h}, 8$ was obtained in 53\% yield $(167 \mathrm{mg})$. Mp: $172-173{ }^{\circ} \mathrm{C}(\mathrm{dec}.) ;{ }^{1} \mathrm{H}$ NMR (300 MHz, $\left.\mathrm{CDCl}_{3}\right): \delta$ 7.32-7.16 (m, 12H), 6.97-6.94 (m, 3H), $5.57(\mathrm{~s}, 1 \mathrm{H}, \mathrm{NH}), 1.89(\mathrm{~s}, 6 \mathrm{H}) ;{ }^{13} \mathrm{C} \mathrm{NMR}(75 \mathrm{MHz}$, $\left.\mathrm{CDCl}_{3}\right): \delta 198.1,143.4,131.8,130.1,129.7,128.9,128.5,126.2,121.8,118.9,118.4,102.6,96.3,77.5$, 11.0; IR $\left(\mathrm{KBr}, \mathrm{cm}^{-1}\right): v(\mathrm{CO})=2026,1970 ; \operatorname{MS}(\mathrm{FAB}): \mathrm{m} / z(\%): 530(12)\left[\mathrm{M}^{+}+1\right], 494(59), 473$ (99), 436 (100). Anal. Calcd for $\mathrm{C}_{27} \mathrm{H}_{22} \mathrm{NO}_{2} \mathrm{ClRu}$ : C, 61.30; H, 4.19; N, 2.65. Found: $\mathrm{C}, 61.22 ; \mathrm{H}, 4.12 ; \mathrm{N}$, 2.82 .

$\left[2,3,4,5-\mathrm{Ph}_{4}\left\{\eta^{5}-\mathrm{C}_{4} \mathrm{CNH}\left(\boldsymbol{p}-\mathrm{NO}_{2} \mathrm{Ph}\right)\right\}\right] \mathrm{Ru}(\mathrm{CO})_{2} \mathrm{Cl}(\mathbf{9})$ : By heating a solution of $\mathrm{Ru}_{3}(\mathrm{CO})_{12}(0.84 \mathrm{~g}, 1.3$ $\mathrm{mmol})$, the imine $(1.00 \mathrm{~g}, 2.0 \mathrm{mmol})$, and dry 2-propanol $(250 \mu \mathrm{L}, 3.0 \mathrm{mmol})$ in chloroform $(26 \mathrm{~mL})$ at 
$90{ }^{\circ} \mathrm{C}$ for 9 days, 9 was obtained in $30 \%$ yield $\left(410 \mathrm{mg}\right.$ ). Mp: $183{ }^{\circ} \mathrm{C}$ (dec.); ${ }^{1} \mathrm{H}$ NMR (300 MHz, $\left.\mathrm{CDCl}_{3}\right): \delta 7.76-6.63(\mathrm{~m}, 24 \mathrm{H}), 6.17(\mathrm{~s}, 1 \mathrm{H}) ;{ }^{13} \mathrm{C} \mathrm{NMR}\left(75 \mathrm{MHz}, \mathrm{CDCl}_{3}\right): \delta$ 197.3, 147.4, 141.7, 132.5, $132.3,129.5,129.2,129.1,128.7,125.4,123.9,118.0,102.9,95.6$; IR $\left(\mathrm{KBr}, \mathrm{cm}^{-1}\right): v(\mathrm{CO})=2042$, 1995; MS (FAB): m/z (\%): 699 (74) $\left[\mathrm{M}^{+}+1\right], 663$ (33), 642 (100), 606 (64). Anal. Calcd for $\mathrm{C}_{37} \mathrm{H}_{25} \mathrm{~N}_{2} \mathrm{O}_{4} \mathrm{ClRu}: \mathrm{C}, 63.66 ; \mathrm{H}, 3.61 ; \mathrm{N}, 4.01$. Found: C, 63.63; H, 3.68; N, 3.97 .

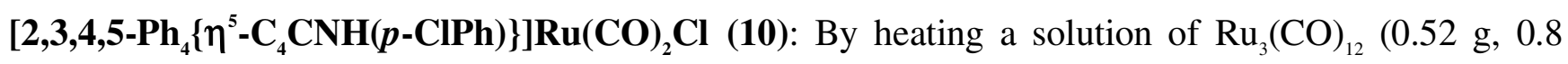
$\mathrm{mmol})$, the imine $(1.00 \mathrm{~g}, 2.0 \mathrm{mmol})$, and dry 2-propanol $(250 \mu \mathrm{L}, 3.0 \mathrm{mmol})$ in chloroform $(26 \mathrm{~mL})$ at $90{ }^{\circ} \mathrm{C}$ for $72 \mathrm{~h}, \mathbf{1 0}$ was obtained in $28 \%$ yield $(404 \mathrm{mg}) . \mathrm{Mp}: 172-173{ }^{\circ} \mathrm{C}(\mathrm{dec}.) ;{ }^{1} \mathrm{H}$ NMR $(300 \mathrm{MHz}$, $\left.\mathrm{CDCl}_{3}\right): \delta 7.29-6.73(\mathrm{~m}, 24 \mathrm{H}), 5.97(\mathrm{~s}, 1 \mathrm{H}) ;{ }^{13} \mathrm{C} \mathrm{NMR}\left(75 \mathrm{MHz}, \mathrm{CDCl}_{3}\right): \delta 198.2,137.0,133.2,132.6$, $130.5,130.3,130.1,129.1,129.0,128.9,128.7,128.4,126.6,102.2,87.3 ; \mathrm{IR}\left(\mathrm{KBr}, \mathrm{cm}^{-1}\right): v(\mathrm{CO})=$ 2031, 1979; MS (FAB): m/z (\%): 688 (1) $\left[\mathrm{M}^{+}+1\right], 652$ (13), 631 (67), 596 (100). Anal. Calcd for $\mathrm{C}_{37} \mathrm{H}_{25} \mathrm{NO}_{2} \mathrm{Cl}_{2} \mathrm{Ru}: \mathrm{C}, 64.63 ; \mathrm{H}, 3.66 ; \mathrm{N}, 2.04$. Found: C, 64.99; H, 3.46; N, 1.92.

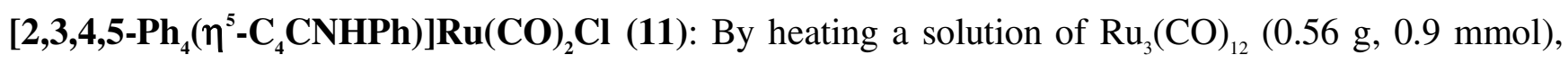
the imine $(1.00 \mathrm{~g}, 1.2 \mathrm{mmol})$, and dry 2-propanol $(150 \mu \mathrm{L}, 1.8 \mathrm{mmol})$ in chloroform $(25 \mathrm{~mL})$ at $90{ }^{\circ} \mathrm{C}$ for $96 \mathrm{~h}, \mathbf{1 1}$ was obtained in $38 \%$ yield $(517 \mathrm{mg})$. Mp: $187-188{ }^{\circ} \mathrm{C}$ (dec.); ${ }^{1} \mathrm{H}$ NMR $\left(300 \mathrm{MHz}, \mathrm{CDCl}_{3}\right)$ : $\delta$ 7.29-6.78 (m, 25H), $6.08(\mathrm{~s}, 1 \mathrm{H}) ;{ }^{13} \mathrm{C} \mathrm{NMR}\left(75 \mathrm{MHz}, \mathrm{CDCl}_{3}\right): \delta 198.4,137.9,136.8,133.0,132.6$, $130.5,130.1,128.9,128.7,128.6,128.3,125.3,124.8,102.0,86.4 ; \operatorname{IR}\left(\mathrm{KBr}, \mathrm{cm}^{-1}\right): v(\mathrm{CO})=2026,1974$; 
MS (FAB): $m / z(\%): 654$ (15) [M+ +1$], 618$ (26), 597 (81), 560 (100). Anal. Calcd for $\mathrm{C}_{37} \mathrm{H}_{26} \mathrm{NO}_{2} \mathrm{ClRu}$ :

C, 68.04; H, 4.01; N, 2.14. Found: C, 68.36; H, 3.82; N, 2.18.

$\left[2,3,4,5-\mathrm{Ph}_{4}\left\{\eta^{5}-\mathrm{C}_{4} \mathrm{CNH}(\boldsymbol{p}-\mathrm{MeOPh})\right\}\right] \mathrm{Ru}(\mathrm{CO})_{2} \mathrm{Cl}(\mathbf{1 2})$ : By heating a solution of $\mathrm{Ru}_{3}(\mathrm{CO})_{12}(0.52 \mathrm{~g}, 0.8$ $\mathrm{mmol})$, the imine $(1.00 \mathrm{~g}, 2.0 \mathrm{mmol})$, and dry 2-propanol $(250 \mu \mathrm{L}, 3.0 \mathrm{mmol})$ in chloroform $(26 \mathrm{~mL})$ at $90{ }^{\circ} \mathrm{C}$ for $48 \mathrm{~h}, 12$ was obtained in $42 \%$ yield $(429 \mathrm{mg})$. Mp: $156-157{ }^{\circ} \mathrm{C}$ (dec.); ${ }^{1} \mathrm{H}$ NMR (300 MHz, $\left.\mathrm{CDCl}_{3}\right): \delta 7.30-6.28(\mathrm{~m}, 24 \mathrm{H}), 6.05(\mathrm{~s}, 1 \mathrm{H}), 3.61(\mathrm{~s}, 3 \mathrm{H}) ;{ }^{13} \mathrm{C} \mathrm{NMR}\left(75 \mathrm{MHz}, \mathrm{CDCl}_{3}\right): \delta 198.3,157.4$, 139.4, 133.1, 132.2, 130.4, 130.0, 128.6, 128.5, 128.3, 128.0, 126.2, 113.7, 101.5, 84.5, 55.8; IR (KBr, $\left.\mathrm{cm}^{-1}\right): \mathrm{v}(\mathrm{CO})=2027,1972$; MS (FAB): m/z (\%): $685(14)\left[\mathrm{M}^{+}+1\right], 650$ (32), 629 (100), 593 (32). Anal. Calcd for $\mathrm{C}_{38} \mathrm{H}_{28} \mathrm{NO}_{3} \mathrm{ClRu}$ : C, 66.81; H, 4.13; N, 2.05. Found: C, 66.49; H, 4.15; N, 1.81 . $\left[\mathbf{2 , 3 , 4 , 5 - \mathrm { Ph } _ { 4 }}{ }_{4}\left[\eta^{5}-\mathrm{C}_{4} \mathbf{C N H}\left(\boldsymbol{p}-\mathrm{NMe}_{2} \mathrm{Ph}\right)\right\}\right] \mathbf{R u}(\mathrm{CO})_{2} \mathbf{C l}(\mathbf{1 3})$ : By heating a solution of $\mathrm{Ru}_{3}(\mathrm{CO})_{12}(0.42 \mathrm{~g}, 0.6$ $\mathrm{mmol})$, the imine $(1.00 \mathrm{~g}, 2.0 \mathrm{mmol})$, and dry 2-propanol $(250 \mu \mathrm{L}, 3.0 \mathrm{mmol})$ in chloroform $(26 \mathrm{~mL})$ at $90{ }^{\circ} \mathrm{C}$ for $48 \mathrm{~h}, \mathbf{1 3}$ was obtained in $9 \%$ yield $(100 \mathrm{mg}) . \mathrm{Mp}: 177-178{ }^{\circ} \mathrm{C}$ (dec.); ${ }^{1} \mathrm{H}$ NMR $(300 \mathrm{MHz}$, $\left.\mathrm{CDCl}_{3}\right): \delta 7.30-6.12(\mathrm{~m}, 24 \mathrm{H}), 6.08(\mathrm{~s}, 1 \mathrm{H}), 2.74(\mathrm{~s}, 6 \mathrm{H}) ;{ }^{13} \mathrm{C} \mathrm{NMR}\left(75 \mathrm{MHz}, \mathrm{CDCl}_{3}\right): \delta 198.7,149.3$, $140.6,133.4,132.6,132.0,130.9,130.6,130.5,129.4,128.8,128.7,128.4,128.3,128.2,127.5,127.3$, 126.5, 113.0, 101.8, 41.5; IR $\left(\mathrm{KBr}, \mathrm{cm}^{-1}\right): v(\mathrm{CO})=2027 ; 1972 ; \mathrm{MS}(\mathrm{FAB}): \mathrm{m} / z(\%): 697(4)\left[\mathrm{M}^{+}+1\right]$, 661 (9), 640 (93), 603 (100). Anal. Calcd for $\mathrm{C}_{39} \mathrm{H}_{31} \mathrm{~N}_{2} \mathrm{O}_{2} \mathrm{ClRu}$ : C, 67.28; H, 4.49 N, 4.02. Found: C, $61.57 ; \mathrm{H}, 3.70 ; \mathrm{N}, 3.90$. 


\section{Characterization of the Chiral Acetates Produced in the DKR of Various Alcohols}

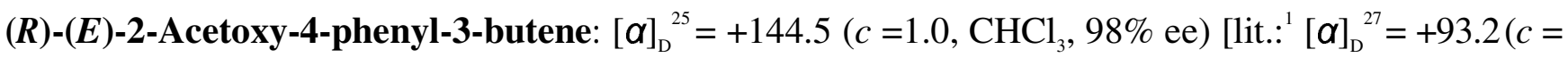
$2.37, \mathrm{CHCl}_{3}, 95 \%$ ee)]; ${ }^{1} \mathrm{H} \mathrm{NMR}\left(300 \mathrm{MHz} \mathrm{CDCl}_{3}\right) \delta 7.40-7.24(\mathrm{~m}, 5 \mathrm{H}), 6.60(\mathrm{~d}, J=16.0 \mathrm{~Hz}, 1 \mathrm{H})$, $6.19\left(\mathrm{dd}, J_{1}=15.9 \mathrm{~Hz}, J_{2}=6.7 \mathrm{~Hz}, 1 \mathrm{H}\right), 5.53(\mathrm{q}, J=6.5 \mathrm{~Hz}, 1 \mathrm{H}), 2.07(\mathrm{~s}, 3 \mathrm{H}), 1.41(\mathrm{~d}, J=6.5 \mathrm{~Hz}, 3 \mathrm{H})$;

${ }^{13} \mathrm{C}$ NMR $\left(75 \mathrm{MHz}, \mathrm{CDCl}_{3}\right) \delta 170.7,136.7,131.9,129.2,128.9,128.3,126.9,71.4,21.8,20.8$; HRMS (EI) $\mathrm{C}_{12} \mathrm{H}_{14} \mathrm{O}_{2}$ calcd $190.0994\left(\mathrm{M}^{+}\right)$, found 190.0990 .

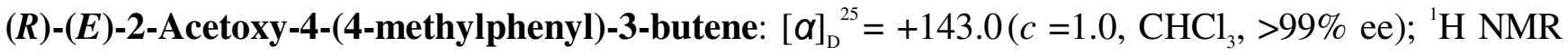
$\left(300 \mathrm{MHz}, \mathrm{CDCl}_{3}\right) \delta 7.27(\mathrm{~d}, J=8.3 \mathrm{~Hz}, 2 \mathrm{H}), 7.12(\mathrm{~d}, J=8.2 \mathrm{~Hz}, 2 \mathrm{H}), 6.57(\mathrm{~d}, J=15.9 \mathrm{~Hz}, 1 \mathrm{H}), 6.13$ $\left(\mathrm{dd}, J_{1}=15.9 \mathrm{~Hz}, J_{2}=6.7 \mathrm{~Hz}, 1 \mathrm{H}\right), 5.52(\mathrm{q}, J=6.5 \mathrm{~Hz}, 1 \mathrm{H}), 2.34(\mathrm{~s}, 3 \mathrm{H}), 2.07(\mathrm{~s}, 3 \mathrm{H}), 1.42(\mathrm{~d}, J=6.5$ $\mathrm{Hz}, 3 \mathrm{H}) ;{ }^{13} \mathrm{C} \mathrm{NMR}\left(75 \mathrm{MHz}, \mathrm{CDCl}_{3}\right) \delta 171.0,138.4,134.2,132.2,129.9,128.4,127.1,71.8,22.1,21.9$, 21.1; HRMS (EI) calcd for $\mathrm{C}_{13} \mathrm{H}_{16} \mathrm{O}_{2}: 204.1150\left(\mathrm{M}^{+}\right)$, found 204.115.

$(\boldsymbol{R})-(\boldsymbol{E})-2-A c e t o x y-4-(4-m e t h o x y p h e n y l)-3-b u t e n e: ~[\alpha]_{\mathrm{D}}{ }^{25}=+137.8\left(c=1.0, \mathrm{CHCl}_{3}, 98.9 \%\right.$ ee); ${ }^{1} \mathrm{H}$ NMR $\left(300 \mathrm{MHz}, \mathrm{CDCl}_{3}\right) \delta 7.28(\mathrm{~d}, J=8.7 \mathrm{~Hz}, 2 \mathrm{H}), 6.81(\mathrm{~d}, J=8.7 \mathrm{~Hz}, 2 \mathrm{H}), 6.53(\mathrm{~d}, J=15.9 \mathrm{~Hz}, 1 \mathrm{H})$, $6.04\left(\mathrm{dd}, J_{1}=15.9 \mathrm{~Hz}, J_{2}=6.9 \mathrm{~Hz}, 1 \mathrm{H}\right), 5.51(\mathrm{q}, J=6.5 \mathrm{~Hz}, 1 \mathrm{H}), 3.80(\mathrm{~s}, 3 \mathrm{H}), 2.06(\mathrm{~s}, 3 \mathrm{H}), 1.39(\mathrm{~d}, J=$ $6.5 \mathrm{~Hz}, 3 \mathrm{H}) ;{ }^{13} \mathrm{C} \mathrm{NMR}\left(75 \mathrm{MHz}, \mathrm{CDCl}_{3}\right) \delta 170.9,160.1,131.8,129.7,128.4,127.2,114.6,71.9,55.9$, 22.1, 21.1; HRMS (EI) $\mathrm{C}_{13} \mathrm{H}_{16} \mathrm{O}_{3}$ calcd $220.1099\left(\mathrm{M}^{+}\right)$, found 220.1095. 
$(\boldsymbol{R})-(\boldsymbol{E})$-2-Acetoxy-4-(4-chlorophenyl)-3-butene: $[\alpha]_{\mathrm{D}}^{25}=+131.8\left(c=1.0, \mathrm{CHCl}_{3}, 98.8 \%\right.$ ee $)\left[\right.$ lit...$^{2}[\alpha$ ]$\left._{\mathrm{D}}^{20}=+139.2\left(c=1.0, \mathrm{CHCl}_{3},>99 \% \mathrm{ee}\right)\right] ;{ }^{1} \mathrm{H} \mathrm{NMR}\left(300 \mathrm{MHz}, \mathrm{CDCl}_{3}\right) \delta 7.32-7.26(\mathrm{~m}, 4 \mathrm{H}), 6.54(\mathrm{~d}, J=$ $16.9 \mathrm{~Hz}, 1 \mathrm{H}), 6.16\left(\mathrm{dd}, J_{1}=15.9 \mathrm{~Hz}, J_{2}=6.7 \mathrm{~Hz}, 1 \mathrm{H}\right), 5.51(\mathrm{q}, J=6.5 \mathrm{~Hz}, 1 \mathrm{H}), 2.07(\mathrm{~s}, 3 \mathrm{H}), 1.40(\mathrm{~d}, J$ $=6.5 \mathrm{~Hz}, 3 \mathrm{H}) ;{ }^{13} \mathrm{C} \mathrm{NMR}\left(75 \mathrm{MHz}, \mathrm{CDCl}_{3}\right) \delta 171.0,135.5,134.2,130.9,130.2,129.4,128.4,71.5$, 22.1, 21.0; HRMS (EI) $\mathrm{C}_{12} \mathrm{H}_{13} \mathrm{ClO}_{2}$ calcd $224.0604\left(\mathrm{M}^{+}\right)$, found 224.0606.

$(\boldsymbol{R})$-(E)-2-Acetoxy-4-(1-naphthyl)-3-butene: $[\alpha]_{\mathrm{D}}{ }^{25}=+71.2\left(c=1.0, \mathrm{CHCl}_{3},>99 \%\right.$ ee); ${ }^{1} \mathrm{H}$ NMR $(300$ $\left.\mathrm{MHz}, \mathrm{CDCl}_{3}\right) \delta 8.10(\mathrm{~d}, J=1.5 \mathrm{~Hz}, 1 \mathrm{H}), 7.83(\mathrm{~d}, J=1.9 \mathrm{~Hz}, 1 \mathrm{H}), 7.78(\mathrm{~d}, J=8.1 \mathrm{~Hz}, 1 \mathrm{H}), 7.59-7.34$ $(\mathrm{m}, 4 \mathrm{H}), 6.21\left(\mathrm{dd}, J_{1}=15.7 \mathrm{~Hz}, J_{2}=6.7 \mathrm{~Hz}, 1 \mathrm{H}\right), 5.64(\mathrm{q}, J=6.5 \mathrm{~Hz}, 1 \mathrm{H}), 2.11(\mathrm{~s}, 3 \mathrm{H}), 1.51(\mathrm{~d}, J=6.5$ $\mathrm{Hz}, 3 \mathrm{H}) ;{ }^{13} \mathrm{C} \mathrm{NMR}\left(75 \mathrm{MHz}, \mathrm{CDCl}_{3}\right) \delta 170.9,134.6,134.0,132.5,131.6,129.3,128.9,128.7,126.6$, 126.3, 125.9, 124.4, 124.2, 71.6, 21.9, 20.9; HRMS (EI) $\mathrm{C}_{16} \mathrm{H}_{16} \mathrm{O}_{2}$ calcd $240.1150\left(\mathrm{M}^{+}\right.$), found 240.1149 .

$(\boldsymbol{R})-(\boldsymbol{E})$-2-Acetoxy-4-(2,4,6-trimethylphenyl)-3-butene: $[\alpha]_{\mathrm{D}}{ }^{25}=+103.1\left(c=1.0, \mathrm{CHCl}_{3},>99 \%\right.$ ee $) ;{ }^{1} \mathrm{H}$ $\operatorname{NMR}\left(300 \mathrm{MHz}, \mathrm{CDCl}_{3}\right) \delta 6.85(\mathrm{bs}, 2 \mathrm{H}), 6.55(\mathrm{~d}, J=16.1 \mathrm{~Hz}, 1 \mathrm{H}), 5.65\left(\mathrm{dd}, J_{1}=16.1 \mathrm{~Hz}, J_{2}=6.8 \mathrm{~Hz}\right.$, $1 \mathrm{H}), 5.51(\mathrm{q}, J=6.5 \mathrm{~Hz}, 1 \mathrm{H}), 2.29(\mathrm{~s}, 9 \mathrm{H}), 2.08(\mathrm{~s}, 3 \mathrm{H}), 1.41(\mathrm{~d}, J=6.5 \mathrm{~Hz}, 3 \mathrm{H}) ;{ }^{13} \mathrm{C}$ NMR $(75 \mathrm{MHz}$, $\left.\mathrm{CDCl}_{3}\right) \delta 171.1,137.1,136.5,134.6,133.9,130.0,129.2,72.2,22.1,21.7,21.5,21.3$; HRMS (EI) $\mathrm{C}_{15} \mathrm{H}_{20} \mathrm{O}_{2}$ calcd 232.1463 $\left(\mathrm{M}^{+}\right)$, found 232.1467. 
$(\boldsymbol{R})$-(E)-2-Acetoxy-4-biphenyl-3-butene: $[\alpha]_{\mathrm{D}}^{25}=+148.6\left(c=1.0, \mathrm{CHCl}_{3},>99 \%\right.$ ee $) ;{ }^{1} \mathrm{H}$ NMR $(300$ $\left.\mathrm{MHz}, \mathrm{CDCl}_{3}\right) \delta$ 7.60-7.57 (m, 3H), 7.56-7.53 (m, 3H), 7.46-7.22 (m, 3H), $6.61(\mathrm{~d}, J=16.0 \mathrm{~Hz}, 1 \mathrm{H})$, 6.26-6.19 (dd, $\left.J_{1}=15.9 \mathrm{~Hz}, J_{2}=6.7 \mathrm{~Hz}, 1 \mathrm{H}\right), 5.54(\mathrm{q}, J=6.5 \mathrm{~Hz}, 1 \mathrm{H}), 2.08(\mathrm{~s}, 3 \mathrm{H}), 1.42(\mathrm{~d}, J=6.5 \mathrm{~Hz}$, $3 \mathrm{H}) ;{ }^{13} \mathrm{C} \mathrm{NMR}\left(75 \mathrm{MHz}, \mathrm{CDCl}_{3}\right) \delta 170.0,141.4,136.1,131.9,130.2,129.6,128.1,127.7,123.6,71.7$, 22.1, 21.7; HRMS (EI) $\mathrm{C}_{18} \mathrm{H}_{18} \mathrm{O}_{2}$ calcd $266.1307\left(\mathrm{M}^{+}\right.$), found 266.1305 .

$(\boldsymbol{R})$-1-Acetoxy-1-cyclohexyl-2-propene: $[\alpha]_{\mathrm{D}}^{25}=+12.8\left(c=1.0, \mathrm{CHCl}_{3},>99 \%\right.$ ee $) ;{ }^{1} \mathrm{H} \mathrm{NMR}(300 \mathrm{MHz}$, $\left.\mathrm{CDCl}_{3}\right) \delta 5.81-5.69(\mathrm{~m}, 1 \mathrm{H}), 5.24-5.17(\mathrm{~m}, 2 \mathrm{H}), 5.07-5.02(\mathrm{t}, J=6.7 \mathrm{~Hz}, 1 \mathrm{H}), 2.07(\mathrm{~s}, 3 \mathrm{H}), 1.81-1.68$ (m, 4H), 1.58-1.48 (m, 1H), 1.29-1.17 (m, 4H), 1.11-0.97 (m, 2H), ${ }^{13} \mathrm{C}$ NMR (75 MHz, $\left.\mathrm{CDCl}_{3}\right) \delta 170.7$, 135.5, 117.8, 79.3, 41.9, 32.0, 28.9, 26.7, 23.0, 21.6, 14.5; HRMS (EI) $\mathrm{C}_{11} \mathrm{H}_{18} \mathrm{O}_{2}$ calcd $182.1307\left(\mathrm{M}^{+}\right)$, found 182.1306 .

$(\boldsymbol{R})-(\boldsymbol{E})$-2-Acetoxy-4-(3-phenoxyphenyl)-3-butene: $[\alpha]_{\mathrm{D}}^{25}=+101.6\left(c=1.0, \mathrm{CHCl}_{3},>99 \%\right.$ ee $) ;{ }^{1} \mathrm{H}$ NMR (300 MHz, $\left.\mathrm{CDCl}_{3}\right) \delta$ 7.36-7.29 (m, 2H), 7.29-7.27 (m, 1H), 7.25-7.23 (m, 2H), 7.13-6.98 (m, $3 \mathrm{H}), 6.91-6.88(\mathrm{~m}, 1 \mathrm{H}), 6.57-6.52(\mathrm{~d}, J=16.0 \mathrm{~Hz}, 1 \mathrm{H}), 6.18-6.11\left(\mathrm{dd}, J_{1}=15.9 \mathrm{~Hz}, J_{2}=6.7 \mathrm{~Hz}, 1 \mathrm{H}\right)$, $5.50(\mathrm{q}, J=6.5 \mathrm{~Hz}, 1 \mathrm{H}), 2.06(\mathrm{~s}, 3 \mathrm{H}), 1.39(\mathrm{~d}, J=6.5 \mathrm{~Hz}, 3 \mathrm{H}) ;{ }^{13} \mathrm{C} \mathrm{NMR}\left(75 \mathrm{MHz}, \mathrm{CDCl}_{3}\right) \delta 170.6$, $157.9,157.6,138.8,131.3,130.2,130.1,126.3,123.6,122.1,119.2,118.8,117.3,71.1,21.7,20.7$; HRMS (EI) $\mathrm{C}_{18} \mathrm{H}_{18} \mathrm{O}_{3}$ calcd $282.1256\left(\mathrm{M}^{+}\right)$, found 282.1256. 
(R)-2-Acetoxy-4-phenyl-3-butyne: $[\alpha]_{\mathrm{D}}{ }^{25}=-153.8\left(c=1.0, \mathrm{CHCl}_{3},>99 \%\right.$ ee $) ;{ }^{1} \mathrm{H}$ NMR $(300 \mathrm{MHz}$, $\left.\mathrm{CDCl}_{3}\right) \delta$ 7.46-7.43 (m, 2H), 7.35-7.28 (m, 3H), 5.72-5.65 (q, $\left.J=6.6 \mathrm{~Hz}, 1 \mathrm{H}\right), 2.11(\mathrm{~s}, 3 \mathrm{H}), 1.58(\mathrm{~d}, J=$ $6.7 \mathrm{~Hz}, 3 \mathrm{H}) ;{ }^{13} \mathrm{C} \mathrm{NMR}\left(75 \mathrm{MHz}, \mathrm{CDCl}_{3}\right) \delta 169.8,131.9,128.5,128.2,122.4,87.5,84.6,60.8,21.5$, 21.0; HRMS (EI) $\mathrm{C}_{12} \mathrm{H}_{12} \mathrm{O}_{2}$ calcd $188.0837\left(\mathrm{M}^{+}\right)$, found 188.0833.

(R)-2-Acetoxy-3-trityloxypropane: $[\alpha]_{\mathrm{D}}{ }^{25}=+29.9\left(c=1.0, \mathrm{CHCl}_{3},>99 \%\right.$ ee); ${ }^{1} \mathrm{H}$ NMR $(300 \mathrm{MHz}$, $\left.\mathrm{CDCl}_{3}\right) \delta$ 7.46-7.24 (m, 15H), 5.17-5.12 (m, 1H), 3.16-3.08 (dd, $\left.J_{1}=14.3 \mathrm{~Hz}, J_{2}=4.1 \mathrm{~Hz}, 2 \mathrm{H}\right), 2.09(\mathrm{~s}$, $3 \mathrm{H}), 1.22(\mathrm{~d}, J=6.5 \mathrm{~Hz}, 3 \mathrm{H}) ;{ }^{13} \mathrm{C} \mathrm{NMR}\left(75 \mathrm{MHz}, \mathrm{CDCl}_{3}\right) \delta 171.3,144.6,129.4,128.5,127.7,87.1$, 70.5, 66.9, 22.0, 17.6; HRMS (EI) $\mathrm{C}_{24} \mathrm{H}_{24} \mathrm{O}_{3}$ calcd $360.1725\left(\mathrm{M}^{+}\right)$, found 360.1723.

(R)-3-Acetoxy-4-trityloxybutane: $[\alpha]_{\mathrm{D}}{ }^{25}=+30.4\left(c=1.0, \mathrm{CHCl}_{3},>99 \%\right.$ ee $) ;{ }^{3}{ }^{1} \mathrm{H}$ NMR $(300 \mathrm{MHz}$, $\left.\mathrm{CDCl}_{3}\right) \delta$ 7.45-7.20 (m, 15H), 5.03-4.99 (m, 1H), 3.13-3.11 (dd, $\left.J_{1}=14.3 \mathrm{~Hz}, J_{2}=4.1 \mathrm{~Hz}, 2 \mathrm{H}\right), 2.10(\mathrm{~s}$, $3 \mathrm{H}), 1.68-1.61(\mathrm{~m}, 2 \mathrm{H}), 0.85-0.80(\mathrm{t}, J=7.5 \mathrm{~Hz}, 3 \mathrm{H}) ;{ }^{13} \mathrm{C} \mathrm{NMR}\left(75 \mathrm{MHz}, \mathrm{CDCl}_{3}\right) \delta 171.5,144.7$, 129.4, 128.5, 127.7, 87.0, 75.1, 65.2, 24.7, 21.9, 10.3; HRMS (EI) $\mathrm{C}_{25} \mathrm{H}_{26} \mathrm{O}_{3}$ calcd $374.1882\left(\mathrm{M}^{+}\right)$, found 374.1885 .

$(\boldsymbol{R})$-2-Acetoxy-4-trityloxybutane: $[\alpha]_{\mathrm{D}}{ }^{25}=-16.3\left(c=1.0, \mathrm{CHCl}_{3},>99 \%\right.$ ee $) ;{ }^{3} \mathrm{H}$ NMR $\left(\mathrm{CDCl}_{3}, 300\right.$ MHz) $\delta$ 7.44-7.19 (m, 15H), 5.16-5.09 (m, 1H), 3.14-3.06 (m, 2H), $1.91(\mathrm{~s}, 3 \mathrm{H}), 1.88-1.79(\mathrm{~m}, 2 \mathrm{H})$, $1.20(\mathrm{~d}, J=6.3 \mathrm{~Hz}, 3 \mathrm{H}) ;{ }^{13} \mathrm{C} \mathrm{NMR}\left(\mathrm{CDCl}_{3}, 75 \mathrm{MHz}\right) \delta 171.1,144.9,129.4,128.4,127.6,126.6,87.3$, 69.3, 60.5, 37.0, 21.9, 20.9; HRMS (EI) $\mathrm{C}_{25} \mathrm{H}_{26} \mathrm{O}_{3}$ calcd $374.1882\left(\mathrm{M}^{+}\right)$, found 374.1880. 
$(\boldsymbol{R}, \boldsymbol{R})-\alpha, \alpha^{\prime}$-Dimethyl-1,3-benzenedimethanol diacetate: $[\alpha]_{\mathrm{D}}{ }^{25}=+147.8(c=1.0$, acetone, $>99 \%$ ee, 97.6\% de) $\left[\right.$ lit.. $.^{4}[\alpha]_{\mathrm{D}}{ }^{25}=+111.3\left(c=2\right.$, acetone, $93 \%$ ee)]; ${ }^{1} \mathrm{H}$ NMR $\left(300 \mathrm{MHz}, \mathrm{CDCl}_{3}\right) \delta$ 7.34-7.27 (m, $4 \mathrm{H}), 5.88(\mathrm{q}, J=6.6 \mathrm{~Hz}, 2 \mathrm{H}), 2.08(\mathrm{~s}, 6 \mathrm{H}), 1.53(\mathrm{~d}, J=6.5 \mathrm{~Hz}, 6 \mathrm{H}) ;{ }^{13} \mathrm{C} \mathrm{NMR}\left(75 \mathrm{MHz}, \mathrm{CDCl}_{3}\right) \delta$ 170.1, 142.0, 128.6, 125.5, 123.9, 72.1, 22.1, 21.2; HRMS (EI) $\mathrm{C}_{14} \mathrm{H}_{18} \mathrm{O}_{4}$ calcd $250.1205\left(\mathrm{M}^{+}\right)$, found 250.1207.

$(\boldsymbol{R}, \boldsymbol{R})-\alpha, \alpha^{\prime}$-Dimethyl-1,4-benzenedimethanol diacetate: $[\alpha]_{\mathrm{D}}^{25}=+165.1(c=1.0$, acetone, $>99 \%$ ee, 96.7\% de) $\left[\right.$ lit.: $.^{4}[\alpha]_{\mathrm{D}}^{25}=+152.9\left(c=2\right.$, acetone, $>97 \%$ ee)]; ${ }^{1} \mathrm{H}$ NMR $\left(300 \mathrm{MHz}, \mathrm{CDCl}_{3}\right) \delta 7.33(\mathrm{bs}, 4 \mathrm{H})$, $5.87(\mathrm{q}, J=6.6 \mathrm{~Hz}, 2 \mathrm{H}), 2.06(\mathrm{~s}, 6 \mathrm{H}), 1.52(\mathrm{~d}, J=6.5 \mathrm{~Hz}, 6 \mathrm{H}) ;{ }^{13} \mathrm{C} \mathrm{NMR}\left(75 \mathrm{MHz}, \mathrm{CDCl}_{3}\right) \delta 170.1$, 141.3, 126.3, 71.9, 22.0, 21.2; HRMS (EI) $\mathrm{C}_{14} \mathrm{H}_{18} \mathrm{O}_{4}$ calcd $250.1205\left(\mathrm{M}^{+}\right)$, found 250.1206.

tert-Butyl- $(\boldsymbol{R})-3$-acetoxybutyrate: $[\alpha]_{\mathrm{D}}^{25}=+7.59\left(c=1.0, \mathrm{CHCl}_{3},>99 \% \text { ee }\right)^{5}\left[\right.$ lit.. $:^{6}[\alpha]_{\mathrm{D}}^{25}=+7.59(c=$ $1.0, \mathrm{CHCl}_{3},>99 \%$ ee)]; ${ }^{1} \mathrm{H} \mathrm{NMR}\left(300 \mathrm{MHz}, \mathrm{CDCl}_{3}\right) \delta 5.23(\mathrm{~m}, 1 \mathrm{H}), 2.47\left(\mathrm{dd}, J_{1}=15.5 \mathrm{~Hz}, J_{2}=7.6 \mathrm{~Hz}\right.$, $1 \mathrm{H}), 2.39\left(\mathrm{dd}, J_{1}=15.5 \mathrm{~Hz}, J_{2}=5.7 \mathrm{~Hz}, 1 \mathrm{H}\right), 1.99(\mathrm{~s}, 3 \mathrm{H}), 1.41(\mathrm{~s}, 9 \mathrm{H}), 1.24(\mathrm{~d}, J=6.3 \mathrm{~Hz}, 3 \mathrm{H}) ;{ }^{13} \mathrm{C}$ NMR (75 MHz, $\left.\mathrm{CDCl}_{3}\right) \delta 170.6,169.9,81.2,68.0,42.6,28.4,21.6,20.2$; HRMS (FAB) $\mathrm{C}_{10} \mathrm{H}_{18} \mathrm{O}_{4}$ calcd $203.1283\left(\mathrm{M}^{+}+1\right)$, found 203.1279.

(S)-1-Acetoxy-1-phenyl-2-chloroethane: $[\alpha]_{D}^{25}=+78.5(c=1.0$, acetone, $95 \%$ ee $)\left[\right.$ lit.: $[\alpha]_{D}^{25}=+77.5$ $\left(c=5.1\right.$, acetone, $97 \%$ ee)]; ${ }^{1} \mathrm{H}$ NMR $\left(300 \mathrm{MHz}, \mathrm{CDCl}_{3}\right) \delta$ 7.38-7.36 (m, 5H), 5.98-5.94 (m, 1H), 3.83- 
$3.69\left(\mathrm{dd}, J_{1}=4.8 \mathrm{~Hz}, J_{2}=11.7 \mathrm{~Hz}, 2 \mathrm{H}\right), 2.13(\mathrm{~s}, 3 \mathrm{H}) ;{ }^{13} \mathrm{C} \mathrm{NMR}\left(75 \mathrm{MHz}, \mathrm{CDCl}_{3}\right) \delta 169.8,137.3,128.7$,

126.7, 75.1, 46.5, 20.9; HRMS (EI) $\mathrm{C}_{10} \mathrm{H}_{11} \mathrm{ClO}_{2}$ calcd $198.0448\left(\mathrm{M}^{+}\right)$, found 198.0443.

(S)-2-Acetoxy-1-phenyloxy-3-chloropropane: $[\alpha]_{\mathrm{D}}{ }^{25}=+20.8\left(c=1.0, \mathrm{CHCl}_{3}, 87 \%\right.$ ee $)$; ${ }^{1} \mathrm{H}$ NMR $(300$ $\left.\mathrm{MHz}, \mathrm{CDCl}_{3}\right) \delta$ 7.32-7.25 (m, 2H), 7.00-6.90 (m, 3H), 5.35-5.32 (q, $\left.J=5.1 \mathrm{~Hz}, 1 \mathrm{H}\right), 4.20-4.16(\mathrm{~m}$, $2 \mathrm{H}), 3.89-3.76\left(\mathrm{dd}, J_{1}=5.2 \mathrm{~Hz}, J_{2}=11.7 \mathrm{~Hz}, 2 \mathrm{H}\right), 2.11(\mathrm{~s}, 3 \mathrm{H}) ;{ }^{13} \mathrm{C} \mathrm{NMR}\left(75 \mathrm{MHz}, \mathrm{CDCl}_{3}\right) \delta 170.1$, 158.4, 129.6, 121.5, 114.8, 71.3, 66.2, 42.5, 20.8; HRMS (EI) $\mathrm{C}_{11} \mathrm{H}_{13} \mathrm{ClO}_{3}$ calcd $228.0553\left(\mathrm{M}^{+}\right)$, found 228.0555.

\section{References}

(1) (a) Midland, M. M.; McDowell, D. C.; Hatch, R. L.; Tramontano, A. J. Am. Chem. Soc. 1980, 102, 867. (b) Burgess, K.; Jennings, L. D. J. Am. Chem. Soc. 1991, 113, 6129.

(2) Brenna, E.; Caraccia, N.; Fuganti, C.; Grasselli, P. Tetrahedron: Asymmetry 1997, 8, 3801.

(3) The \% ee value was determined for the hydrolyzed product by HPLC on a Chiralcel OD.

(4) Wallace, J. S.; Baldwin, B. W.; Morrow, C. J. J. Org. Chem. 1992, 57, 5231.

(5) The $\%$ ee value was determined of the benzoylated derivative, which was obtained through the hydrolysis of the acetylated product, followed by the benzoylation with benzoyl chloride, by HPLC on a $(R, R)$-Whelk-O1. 
(6) Kim, M.-J.; Choi, Y. K.; Choi, M. Y.; Park, J. J. Org. Chem. 2001, 66, 4736.

(7) Hiratake, J.; Inagaki, M.; Nishioka,T.; Oda, J. J. Org. Chem. 1988, 53, 6130. 